\title{
Perspective Silhouette of a General Swept Volume
}

\author{
Joon-Kyung Seong ${ }^{a}$ Ku-Jin Kim ${ }^{b}$ Myung-Soo Kim ${ }^{a}$ \\ Gershon Elber ${ }^{c}$ \\ ${ }^{a}$ Seoul National University, KOREA \\ ${ }^{b}$ Kyungpook National University, KOREA \\ ${ }^{c}$ Technion-Israel Institute of Technology, ISRAEL
}

\begin{abstract}
We present an efficient and robust algorithm for computing the perspective silhouette on the boundary of a general swept volume. We also construct the topology of connected components of the silhouette. As a three-dimensional object moves along a trajectory, each instance of the moving object touches the envelope surface of the swept volume along a characteristic curve $K^{t}-$ a curve $K$ at time $t$. Moreover, the same instance of the moving object has its silhouette curve $L^{t}$ on its own boundary. The intersection $K^{t} \cap L^{t}$ contributes to the silhouette of the general swept volume. We reformulate the problem as a system of two polynomial equations in three variables. Moreover, connected components of a silhouette curve are constructed by detecting the cases where the two curves $K^{t}$ and $L^{t}$ intersect tangentially each other on the boundary surface of the moving object.
\end{abstract}

\section{Introduction}

To give an artistic impression or to draw a conceptual image of a geometric object, it is common to use line drawing techniques, such as pencil, pen-ink drawing or hatching lines. Non-photorealistic rendering is thus quite popular in computer graphics [5]. Automatic generation of artistic curves or lines is an important research topic in this area. Silhouettes are among the most important lines in describing the shape of a three-dimensional object. Computation of silhouette curves is needed in constructing the visible area of an object, removing hidden curves, and performing backface culling, to mention only a few. Since silhouettes are view dependent, they should be reconstructed at each frame of an animation. Furthermore, stylizing silhouettes requires silhouette lines to be connected so as to simulate an artistic

\footnotetext{
${ }^{1}$ Email: swallow@3map.snu.ac.kr (Joon-Kyung Seong).
}

effect of drawing long smooth strokes. It is a non-trivial task to accomplish all these requirements in an efficient way.

In this paper, we present an efficient algorithm for computing the silhouette curves on the boundary of a general swept volume. Given a three-dimensional object $O$, moving under a continuous affine transformation $A(t)$, its swept volume is defined as $\cup_{t} A(t)[O]$. Sweeps are widely accepted as an effective design tool for creating highly complex three-dimensional shapes [1].

Joy and Duchaineau [8] compute the boundary of a swept volume using a Marching Cube algorithm in the $x y z$-space. Kim and Elber [10] reformulate the problem as a polynomial equation in three variables, which is considerably easier to compute. In either case, the boundary surface of a swept volume is approximated by polygons. The silhouette curves are then approximated by line segments. Instead of using two steps, it is more efficient to generate the silhouette curves in a single step.

There has been considerable research on developing efficient algorithms for computing the silhouettes of polyhedral models (see Isenberg et al. [6] for a recent survey). The topological structure of silhouette curves is important not only for a correct rendering but also for the analysis of the shape. For a coherent stylizing of an animated object, the topological change of its silhouette plays an important role. In a recent work, Elber et al. [4] analyzed the topological structure of silhouette curves, and they solve the 2-piece mold separability problem in manufacturing processes such as injection molding or die casting by using the topology information. In computer vision, the topology of silhouettes has been investigated for the construction of the aspect graph [2], a structure that provides all topologically distinct silhouette configurations. Aspect graphs were mainly constructed for polyhedral models. Kim and Lee [9] presented an algorithm for computing the silhouette of a canal surface. In the current paper, we extend this result to the general 
swept volume.

At a fixed time $t$, the transformed object $A(t)[O]$ touches the envelope boundary surface along a characteristic curve, $K^{t}$. Moreover, let $L^{t}$ denote the silhouette curve of this object from a view point $\vec{P}$ (see Figure 1). The union of the intersection points $K^{t} \cap L^{t}$ generates the silhouette curves on the boundary of the swept volume. $K^{t}$ and $L^{t}$ are curves located on the boundary surface of the moving object $A(t)[O]$. The number of intersection points changes through tangential intersections between $K^{t}$ and $L^{t}$. A new loop of the silhouette curve may start when the two curves $K^{t}$ and $L^{t}$ intersect tangentially; or the construction of a closed loop may be completed at such a tangential intersection. Thus the tangential intersections between $K^{t}$ and $L^{t}$ are critical events in the silhouette construction.

The main contribution of our work can be summarized as follows:

- We generate the silhouette curves on the boundary of a general swept volume;

- We construct the topology of connected components of the silhouette curves.

The rest of this paper is organized as follows. In Section 2 , we discuss the extraction of silhouette curves. Section 3 considers the topological structure of the silhouette curves. Experimental results are presented in Section 4. Finally, in Section 5, we conclude the paper.

\section{Extraction of the Silhouette Curves}

In this section, we compute the perspective silhouette curves on the boundary of a general swept volume. We reduce the problem to that of solving two polynomial equations in three variables.

Let $O$ denote a three-dimensional object bounded by a rational parametric freeform surface $S(u, v)$, and let $A(t)$ denote a continuous affine transformation. The swept volume of the object $O$ under the affine transformation $A(t)$ is given as $\cup_{t} A(t)[O]$. Assuming $a \leq t \leq b$, the boundary surface of the swept volume consists of some patches of $A(a)[S(u, v)]$ and $A(b)[S(u, v)]$, together with the boundary envelope surface. The set of points on the envelope surface is characterized by the following equation [10][11]:

$$
\begin{aligned}
F & (u, v, t) \\
& =\left|A^{\prime}(t)[S(u, v)] A(t)\left[\frac{\partial S}{\partial u}(u, v)\right] A(t)\left[\frac{\partial S}{\partial v}(u, v)\right]\right| \\
& =0 .
\end{aligned}
$$

That is, the Jacobian of the trivariate volume $A(t)[S(u, v)]$ vanishes on the envelope surface. Having a single constraint in three variables, the solution set is a 2-manifold in a threedimensional space.

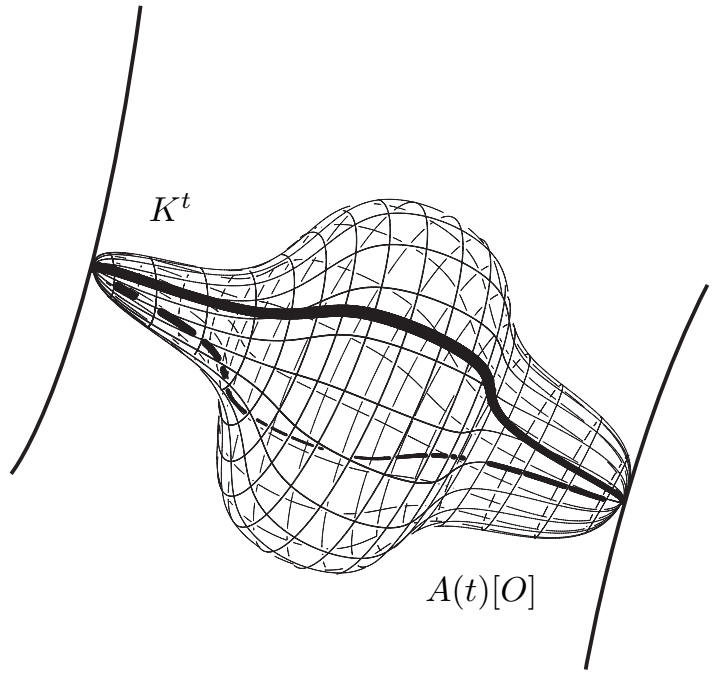

(a)

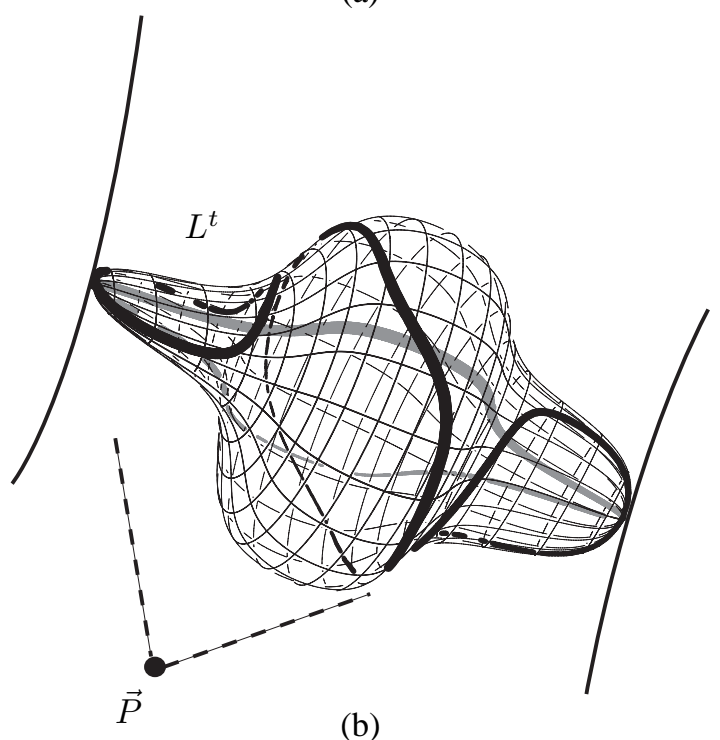

Figure 1: (a) When an object $O$ moves under a continuous affine transformation $A(t)$, the characteristic curve $K^{t}$ (in bold lines) touches the boundary envelope surface; (b) The silhouette curve $L^{t}$ from a viewpoint $\vec{P}$ is shown in bold lines together with $K^{t}$ shown in gray.

The silhouette points on the boundary of the swept volume $A(t)[S(u, v)]$ from a view point $\vec{P}$ satisfies the following implicit equation:

$$
\begin{aligned}
& G(u, v, t) \\
& \quad=\langle A(t)[S(u, v)]-\vec{P}, A(t)[N(u, v)]\rangle=0,
\end{aligned}
$$

where $N(u, v)$ is the normal of $S(u, v)$. Since $N(u, v)=$ $\frac{\partial S}{d u} \times \frac{\partial S}{d v}$ is rational, the function $G(u, v, t)$ is also rational. The common zero-set of Equations (1) and (2) produces 1manifold curves in the uvt-space, which correspond to the silhouette curves on the boundary of the swept volume.

Since $F(u, v, t)=0$ and $G(u, v, t)=0$ are rational equations, their common zero-set can be computed in a highly 
robust manner using the convex hull and subdivision properties of rational spline functions. The computation procedure is reasonably efficient. Solving two equations in three variables, the result is a univariate curve in the $u v t$-space, which can be parameterized by a variable $s$ :

$$
(u(s), v(s), t(s)) .
$$

See Elber and Kim [3] or Patrikalakis and Maekawa [12] for more details of how to solve a system of $m$ polynomial equations in $n$ variables.

\section{Topology of the Silhouette Curves}

In the previous section, we reduced the silhouette construction to a problem of computing the common zero-set of two polynomial equations in three variables. Now we consider how to determine the topological structure of the silhouette curves. For this purpose, we present an algorithm for constructing each connected component of the silhouette curve.

Consider a point $(u, v, t)$ in the common zero-set of Equations (1) and (2). The physical meaning of $F(u, v, t)=0$ is that the boundary surface $A(t)[S(u, v)]$ of a moving object $A(t)[O]$ touches the boundary envelope surface of a swept volume $\cup_{t} A(t)[O]$ along a characteristic curve $K^{t}$. Moreover, the condition $G(u, v, t)=0$ implies that the surface point $A(t)[S(u, v)]$ is on the silhouette curve $L^{t}$ on the boundary of the moving object $A(t)[O]$. Figure 1 shows the characteristic curve $K^{t}$ and the silhouette curve $L^{t}$ of a moving object $A(t)[O]$ in bold lines. Under a continuous affine transformation $A(t)$, the intersection points in the set $K^{t} \cap L^{t}$ trace out the whole silhouette curve on the boundary of the swept volume.

Now we consider a connected component $(u(s), v(s), t(s))$, $\left(s_{0} \leq s \leq s_{1}\right)$, in the common zero-set of $F(u, v, t)=$ $G(u, v, t)=0$. Either it forms a closed loop or it has an endpoint at $t=0$ or $t=1$ (See Figure 3). In the case of a closed loop, there are at least two $t$-extreme points $(u, v, t)$ on the loop, which can be computed by solving the following system of three equations in three variables:

$$
\begin{aligned}
& F(u, v, t)=0 \\
& G(u, v, t)=0 \\
& H(u, v, t)=F_{u} G_{v}-F_{v} G_{u}=0
\end{aligned}
$$

where $F_{u}, F_{v}, G_{u}, G_{v}$ are partial derivative of $F$ and $G$. The physical meaning of a $t$-extreme point is that the two curves $K^{t}$ and $L^{t}$ touch each other tangentially on the boundary surface of $A(t)[O]$. (See the two curves at $t=t_{i-1}, t_{i}$ in Figure 2.) The other case of having an endpoint at $t=0,1$ can be handled by using the solutions $(u, v, t)$ such that $t=0,1$ among the common zeros of Equations (1) and (2).

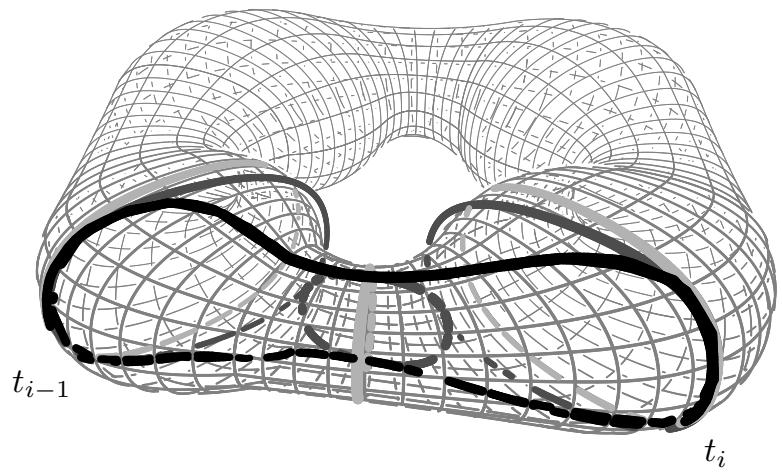

Figure 2: $K^{t}$ is shown in light gray lines and $L^{t}$ is in dark gray lines for $t_{i-1} \leq t \leq t_{i}$. A silhouette curve in bold black lines which is the union of $K^{t} \cap L^{t}, \forall t \in\left[t_{i-1}, t_{i}\right]$.

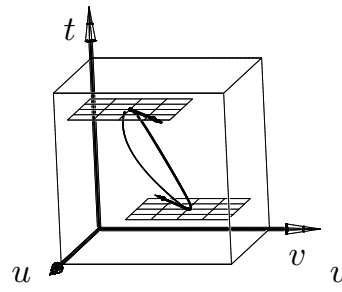

(a)

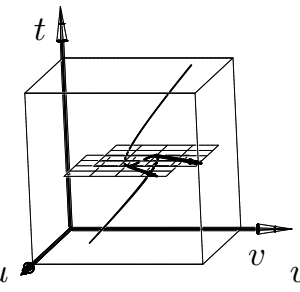

(b)

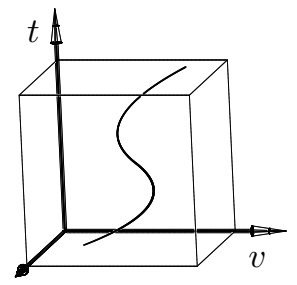

(c)
Figure 3: A classification into three curve types which determines the topology of the silhouette curves: (a) a loop, (b) a curve with local extrema, and (c) a curve that is $t$ monotone. The outlined box represents the domain of the parameter space.

The simultaneous solutions of Equations (1)-(3) correspond to all $t$-extreme points in the common zero-set of $F(u, v, t)=G(u, v, t)=0$. These include local $t$-extreme points as well. Figure 3 shows three typical types of connected components in the common zero-set. Figure 3(c) shows a connected component with no local $t$-extreme point; but this component has both ends at $t=0,1$. We define three different types of connected components:

- A component of Type1 is a closed loop (Figure3(a)).

- A component of Type 2 has some local $t$-extreme points (Figure3(b)).

- A component of Type3 has no local $t$-extreme point. It is $t$-monotone (Figure3(c)).

Connected components are constructed by numerically tracing the intersection curve $F(u, v, t)=G(u, v, t)=0$, starting from $t$-extreme points or endpoints with $t=0,1$. Algorithm 1 summarizes the whole procedure of constructing the silhouette curve.

\section{Experimental Results}

We now present a few examples of computing silhouette curves on the boundary of a general swept volume. Fig- 


\section{Algorithm 1}

Input:

$S(u, v)$, A rational freeform surface;

$A(t)$, An affine transformation matrix;

Output:

$\vec{P}$, The eye position;

A set of perspective silhouette curves of

\section{Begin} $A(t)[S(u, v)]$ from $\vec{P}$

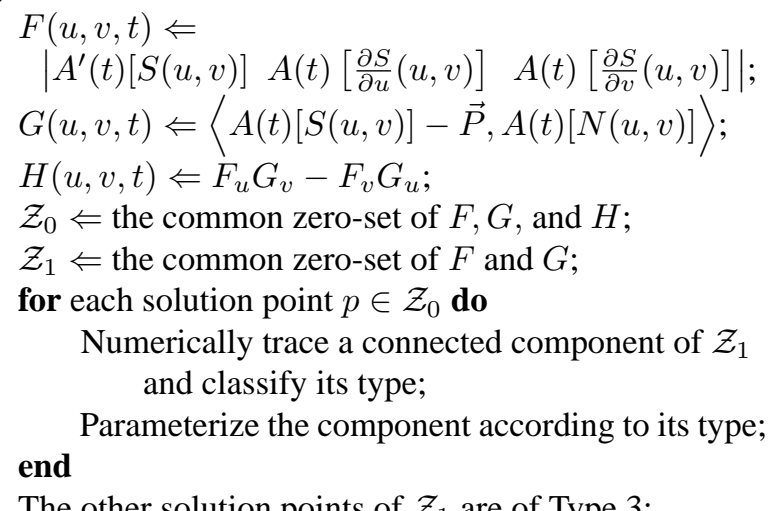

The other solution points of $\mathcal{Z}_{1}$ are of Type 3 ;

Numerically trace each component starting from the solutions of $F(u, v, t)=G(u, v, t)=0$ where $t=0,1$;

return A set of silhouette curves;

End.

ure 4 shows the swept volume of an ellipsoid moving along a linear trajectory under scale change. Its perspective silhouette curves are shown in bold lines (Figure 4 (b)). They are both of type 2. Figure 4(c) shows the projection of the zero-set on to the $v t$-plane. Two more examples are shown in Figure 5. The silhouette curves are applied to nonphotorealistic rendering of the boundary envelope surfaces of three-dimensional swept volumes. The execution time for these examples took one or two seconds on a modern desktop PC.

\section{Conclusion}

In this paper, we have presented a robust and efficient method for computing the perspective silhouette of a general swept volume. We computed the silhouette curves on the boundary envelope surface and also detected all connected components of the silhouette. The silhouette computation problem was reduced to solving a system of two polynomial equations in three variables. Connected components of the silhouette curve was detected and constructed using $t$-extreme points in the common zero-set.

Our algorithm can easily be extended to a general case where the eye position, $\vec{p}(r)$, moves along a predefined path.
In this case, Equations (1) and (2) are given in four variables, and they prodecue a 2-manifold zero-set. The $r$ extreme points on the 2-manifold characterize critical events where a silhouette component may appear or disappear. For this purpose, we need to add two additional polynomial equations. We will investigate more details of this approach in the future work.

\section{REFERENCES}

[1] Alias-Wavefront Technology. 2003. Maya 5.0 Users Manual. http://www.alias.com.

[2] R. Cipolla and P. Giblin. Visual Motion of Curves and Surfaces. Cambridge University Press. 2000.

[3] G. Elber and M.-S. Kim. Geometric constraint solver using multivariate rational spline functions. Proc. of ACM symposium on Solid Modeling and Applications, Ann Arbor, MI, June 4-8, 2001.

[4] G. Elber, X. Chen, and E. Cohen. Mold Accessibility via Gauss Map Analysis. SMI04, Genova, Italy, pp. 263-272, June 2004.

[5] B. Gooch and A. Gooch. Non-Photorealistic Rendering. A.K. Peters Ltd. Publishers ISBN: 1-56881-133$0,2001$.

[6] T. Isenberg, B. Freudenberg, N. Halper, S. Schlechtweg, and T. Strothotte. A developer's guide to silhouette algorithms for polygonal models. IEEE $C G \& A$, pp. 28-37, 2003.

[7] IRIT 9.0 User's Manual, October 2002, Technion. http://www.cs.technion.ac.il/ irit.

[8] K. Joy and M. Duchaineau. Boundary determination for trivariate solid. Proc. of Pacific Graphics 99, Seoul, Korea, pp. 82-91, October 5-7 1999.

[9] K.-J. Kim and I.-K. Lee. The Perspective Silhouette of a Canal Surface. Computer Graphics Forum, Vol. 22, Number 1, pp. 15-22, 2003.

[10] M.-S. Kim and G. Elber. Problem reduction to parameter space. the Mathematics of Surfaces IX (Proc. of the Ninth IMA Conference), R. Cipolla and R. Martin(eds), Springer, London, pp. 82-98, 2000.

[11] R. Martin and P. Stephenson. Sweeping of ThreeDimensional Objects. Computer-Aided Design, Vol. 22, pp. 223-234, 1990.

[12] N. Patrikalakis and T. Maekawa. Shape Interrogation for Computer Aided Design and Manufacturing. Springer, 2002. 


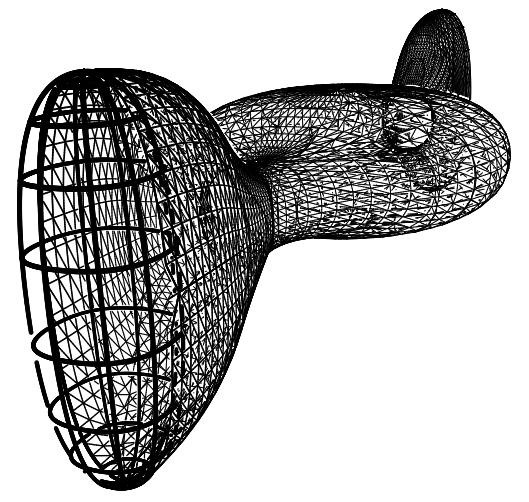

(a)

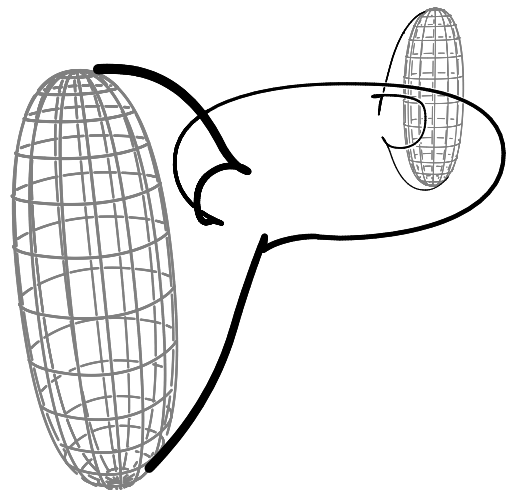

(b)

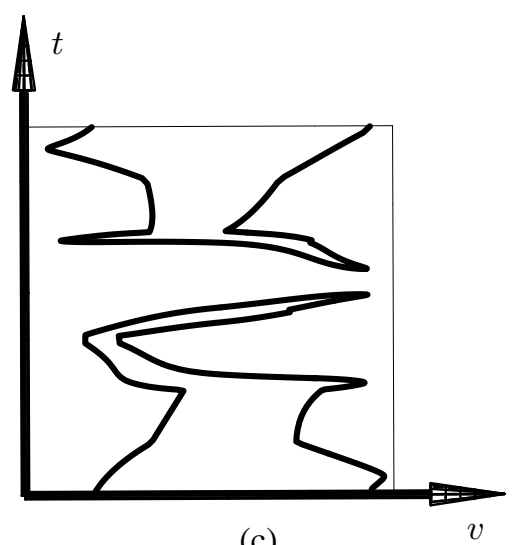

(c)

Figure 4: (a) The envelope of a scaled ellipsoid along a linear trajectory and (b) its perspective silhouette curves. The silhouette curves in the $v t$-space is shown in (c). Two curve components of Type 2 are detected.

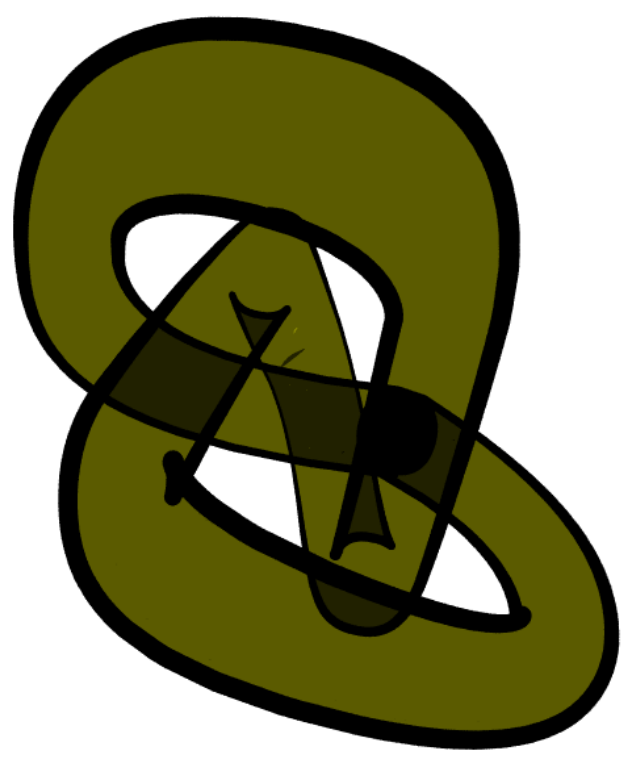

(a)

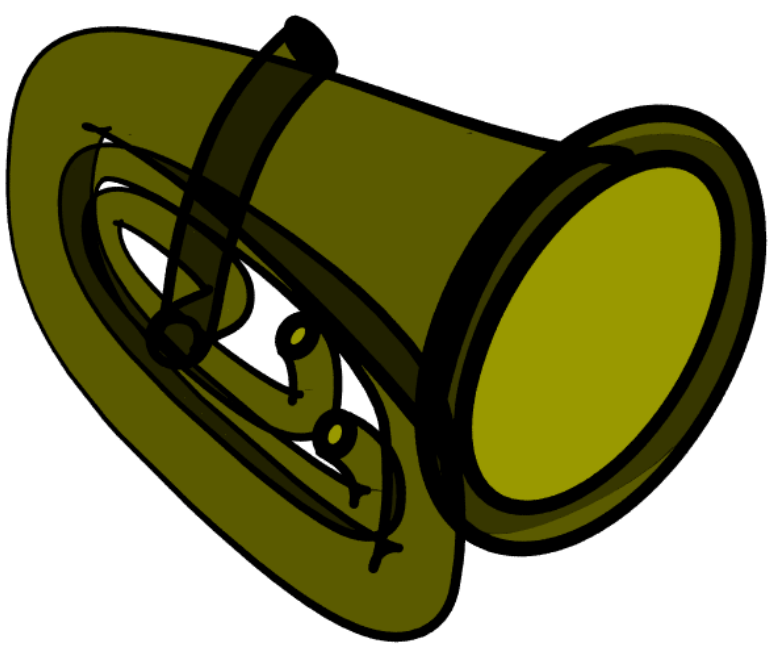

(b)

Figure 5: (a) The envelope surface and its silhouette curves are shown in bold lines for a swept volume of an ellipsoid moving along along a trajectory with scale change. (b) A tuba is modeled by sweeping a sphere and a torus. 